\title{
Single-cultivar extra virgin olive oil classification using a potentiometric electronic tongue
}

\author{
Luís G. Dias a ${ }^{\text {, Andreia Fernandes }}{ }^{\mathrm{a}}$, Ana C.A. Veloso ${ }^{\mathrm{b}, \mathrm{c}}$, Adélio A.S.C. Machado ${ }^{\mathrm{d}}$, José A. Pereira ${ }^{\mathrm{a}}$, \\ António M. Peres ${ }^{\mathrm{a}, \mathrm{e}, *}$ \\ ${ }^{a}$ CIMO - Mountain Research Centre, Escola Superior Agrária, Instituto Politécnico de Bragança, Campus Santa Apolónia, Apartado 1172, $5301-855$ Bragança, Portugal \\ ${ }^{\mathrm{b}}$ Instituto Politécnico de Coimbra, ISEC, DEQB, Rua Pedro Nunes, Quinta da Nora, 3030-199 Coimbra, Portugal \\ ${ }^{\mathrm{C}} \mathrm{CEB}$ - Center of Biological Engineering, University of Minho, Campus de Gualtar, 4710-057 Braga, Portugal \\ d LAQUIPAI - Laboratório de Química Inorgânica Pura e de Aplicação Interdisciplinar, Departamento de Química, Faculdade de Ciências da, Universidade do Porto, \\ Rua Campo Alegre no. 687, 4169-007 Porto, Portugal \\ e LSRE - Laboratory of Separation and Reaction Engineering - Associate Laboratory LSRE/LCM, Escola Superior Agrária, Instituto Politécnico de Bragança, \\ Campus Santa Apolónia, Apartado 1172, 5301-855 Bragança, Portugal
}

\section{A R T I C L E I N F O}

\section{Article history:}

Received 23 January 2014

Received in revised form 4 March 2014

Accepted 12 March 2014

Available online 29 March 2014

\section{Keywords:}

Extra virgin olive oil

Single olive cultivar

Potentiometric electronic tongue

Linear discriminant analysis

Simulated annealing algorithm

\begin{abstract}
A B S T R A C T
Label authentication of monovarietal extra virgin olive oils is of great importance. A novel approach based on a potentiometric electronic tongue is proposed to classify oils obtained from single olive cultivars (Portuguese cvs. Cobrançosa, Madural, Verdeal Transmontana; Spanish cvs. Arbequina, Hojiblanca, Picual). A meta-heuristic simulated annealing algorithm was applied to select the most informative sets of sensors to establish predictive linear discriminant models. Olive oils were correctly classified according to olive cultivar (sensitivities greater than 97\%) and each Spanish olive oil was satisfactorily discriminated from the Portuguese ones with the exception of cv. Arbequina (sensitivities from $61 \%$ to $98 \%$ ). Also, the discriminant ability was related to the polar compounds contents of olive oils and so, indirectly, with organoleptic properties like bitterness, astringency or pungency. Therefore the proposed E-tongue can be foreseen as a useful auxiliary tool for trained sensory panels for the classification of monovarietal extra virgin olive oils.
\end{abstract}

(c) 2014 Elsevier Ltd. All rights reserved.

\section{Introduction}

Olive oils may be classified according to their quality, in a decreasing order of both quality and price, as extra virgin olive oils (EVOO), virgin olive oils (VOO) or lampante olive oils (LOO) (Cano et al., 2011; Dais, \& Hatzakis, 2013; Garcia, Martins, \& Cabrita, 2013; García-González, \& Aparicio, 2004). Some high quality and expensive olive oils (EVOO and VOO) are certified as Protected Denomination of Origin (PDO), which among other features are related to olive oil production and processing made in a specific geographical origin (Casale, Casolino, Oliveri, \& Forina, 2010; Cosio, Ballabio, Benedetti, \& Gigliotti, 2006; Haddi et al., 2011; Haddi et al., 2013; Karabagias, Michos, Badeka, Kontakos, \& Kontominas, 2013; Montealegre, Alegre, \& Garcia-Ruíz, 2010; Pizarro,

\footnotetext{
* Corresponding author at: LSRE - Laboratory of Separation and Reaction Engineering - Associate Laboratory LSRE/LCM, Escola Superior Agrária, Instituto Politécnico de Bragança, Campus Santa Apolónia, Apartado 1172, 5301-855 Bragança, Portugal. Tel.: +351 273303220; fax: +351 273325405.

E-mail address: peres@ipb.pt (A.M. Peres).
}

Rodríguez-Tecedor, Pérez-del-Notario, Esteban-Díez, \& González-Sáiz, 2013). Recently, extra emphasis has been given to the botanical origin of olive oils, due to the marketing of high-quality and high-price monovarietal hallmark EVOO (Cimato et al., 2006; Cosio et al., 2006; Garcia et al., 2013; Matos et al., 2007; Montealegre et al., 2010; Ruiz-Samblás et al., 2012). Label information regarding region of origin affects product acceptability, while information about cultivar significantly affects the expectation of bitterness and pungency for olive oils (Delgado, Gómez-Rico, \& Guinard, 2013). Indeed, consumers' preference is changing towards food products with certified genuineness and geographical origin (Cosio et al., 2006). EVOO are high-price food products, highly appreciated and an important component of the Mediterranean diet (Ruiz-Samblás et al., 2012). Their quality and uniqueness is attributed to several factors like cultivar or varietal olive origin, environment, crop season, degree of maturation and cultural practises (Bakhouche et al., 2013; Cosio et al., 2006; Longobardi et al., 2012). Therefore, EVOO are one of the food products most prone to frauds that mainly involve mislabelling, adulteration or mixing with cheaper oils (Moore, Spink, \& Lipp, 2012; Nunes, 2013; Pizarro et al., 2013). 
Several official methods and recommended practises have been established by the American Oil Chemists' Society to assess oil quality (Nunes, 2013). However, some of these methods are quite expensive, non-green techniques, and require fulfilment of straight standardised procedures to ensure accuracy. Therefore, other approaches have been reported trying to overcome some of these drawbacks, namely gas-, liquid-chromatography and, more recently, mass-spectrometry based-methodologies (Bakhouche et al., 2013; Garcia et al., 2013; Garrido-Delgado et al., 2011; Karabagias et al., 2013; Lauri et al., 2013; Longobardi et al., 2012; López-Feria, Cárdenas, García-Mesa, \& Valcárcel, 2008; Matos et al., 2007; Montealegre et al., 2010; Romero, \& Brenes, 2012; Ruiz-Samblás et al., 2012). These are based on the identification and quantification of several chemical olive oil components that allow assessment of the quality, authenticity or adulteration of olive oils. Also, DNA-based methods have been proposed to authenticate the varietal origin of olive oils (Bazakos et al., 2012), and non-destructive spectroscopy-based methods have been used to evaluate olive oils quality, authenticity and possible adulterations (Dais, \& Hatzakis, 2013; Nunes, 2013; Pizarro et al., 2013). Nevertheless, these approaches require highly-skilled technicians and usually are not suitable for in situ applications, being far beyond the economic possibilities of small producers or retailers.

Therefore, low-cost, portable, fast, accurate, reliable and robust analytical methodologies are still needed for authentication purposes and routine analysis. The potential use of electrochemical sensors to evaluate olive oil quality and their application in authentication or adulteration assays can be seen as a possible alternative tool. Indeed, electronic noses (E-noses) and electronic tongues (E-tongues), individually or combined, have been proposed in the last decade for olive oil characterisation (Ruiz-Samblás et al., 2012) using different multivariate statistical techniques. E-noses have been successfully used to classify and discriminate EVOO, VOO and LOO (Escuderos, Sánchez, \& Jiménez, 2010; Escuderos, Sánchez, \& Jiménez, 2011; García-González, \& Aparicio, 2004); to classify VOO according to their geographical origin (Haddi et al., 2011), and to differentiate single-cultivar or multi-cultivar EVOO according to Protected Designation of Origin (Cimato et al., 2006). Voltammetric E-tongues have been applied with success to separate EVOO, VOO, LOO and refined olive oils (Apetrei, \& Apetrei, 2013; Apetrei, Rodríguez-Méndez, \& de Saja, 2005; Oliveri, Baldo, Daniele, \& Forina, 2009) and to distinguish EVOO from maize oils, different EVOO from the same geographical region or EVOO from different countries (Oliveri et al., 2009). Hybrid systems, combining E-noses and voltammetric E-tongues, were proposed and effectively applied to discriminate monovarietal or multivarietal EVOO or VOO according to their geographical origin (Apetrei et al., 2010; Cosio et al., 2006; Haddi et al., 2013). Among these works on the application of electrochemical methodologies for olive oils analysis, only one deals with the possibility of differentiating monovarietal EVOO according to the olive cultivar: Cimato et al. (2006) applied an E-nose to classify samples of 12 Italian single-cultivar EVOO but the results showed that it was only possible to separate among clusters of different monovarietal EVOO. These electrochemical devices have also been applied to evaluate olive oil chemical composition changes during storage under different conditions, namely temperature, light and storage time, showing that those conditions clear influence olive oil organoleptic characteristics, namely the polyphenols composition of VOO due to lipid-radical reactions or to enzymatic activity (Clodoveo, Hbaieb, Kotti, Mugnozza, \& Gargouri, 2014; Cosio, Ballabio, Benedetti, \& Gigliotti, 2007; Lerma-García, Simó-Alfonso, Bendini, \& Cerretan, 2009).

These satisfactory results for olive oil analysis using electrochemical sensors have stimulated the present work. In this study, and to the authors' best knowledge, a potentiometric E-tongue with cross-sensitivity non-specific lipidic sensor membranes is proposed for the first time to discriminate Portuguese (PT) and Spanish (ES) monovarietal EVOO according to the olive cultivar (cv. Cobrançosa (COB), cv. Madural (MAD) or cv. Verdeal Transmontana (VER); and cv. Arbequina (ARB), cv. Hojiblanca (HOJ) or cv. Picual (PIC), respectively). Furthermore, for the first time ever an electrochemical approach is applied to the analysis of PT monovarietal EVOO. To establish linear discriminant models with the best cross-validation predictive performance, the most informative potentiometric sensor signal profiles obtained during EVOO analysis were selected using a simulated annealing (SA) algorithm, which is a meta-heuristic variable selection algorithm. Due to the difficulty of carrying out electrochemical assays in non-conductive liquids with high viscosity (Apetrei et al., 2010) in the present work, hydro-ethanolic extracts of EVOO were used. Previously, the use of hydro-methanolic mixtures was already reported to overcome this issue (Rodríguez-Méndez, Apetrei, \& de Saja, 2008). These alcoholic extracts are expected to be rich in polar compounds, such as sterols, polyphenols and tocopherols, which differ considerably among olive oils cultivars and are the main contributors to olive oil bitterness, astringency and pungency (Apetrei et al., 2010; Carrasco-Pancorbo et al., 2006; García, Brenes, Garcí, Romero, \& Garrido, 2003; García, Brenes, Romero, García, \& Garrido, 2002; Matos et al., 2007; Morelló, Romero, \& Motilva, 2004; Romero, \& Brenes, 2012). Therefore their use as suitable discriminating descriptors was investigated, since it is expected that those compounds (e.g., sterols, polyphenols and tocopherols) are the major constituents of the hydro-ethanolic extracts. The three PT cultivars were chosen due to their economic importance since they account for more than $90 \%$ of the olive cultivation area in Trás-os-Montes region (northeast of Portugal) and can be used to produce PDO "Trás-os-Montes olive oil" (Matos et al., 2007). The three ES cultivars were selected since they are the most common varieties cultivated in Spain (Ruíz-Samblás et al., 2012) and recently they have been introduced in northeast PT olive groves due to their high productivity and easy adaptation to the edaphoclimatic conditions.

\section{Materials and methods}

\subsection{Extra-virgin olive oil samples}

The PT and ES EVOO of different olive cultivars (cvs. Cobrançosa, Madural, Verdeal Transmontana; and Arbequina, Hojiblanca, Picual, respectively), produced in the northeast of Portugal (Mirandela and Valpaços in the Trás-os-Montes region) and the north of Spain (Valladolid region), were studied. Eighteen samples of monovarietal EVOO were obtained directly from certified olive oil producers. Details regarding olive oil variety, geographical origin and year of production are given in Table 1. All olive oil samples analysed were packed and stored in the dark at $-20^{\circ} \mathrm{C}$ in a 24 -h period after their production in olive mills with a two-phase extraction process (in 2011 and 2012), and kept under those conditions until further analysis. All samples were electrochemically analysed on the same day, equivalent to storage times of one year or less.

\subsection{Extra-virgin olive oil extraction procedure}

Polar compounds from each monovarietal EVOO were extracted using a hydroethanolic solution $\left(\mathrm{H}_{2} \mathrm{O}: \mathrm{EtOH}, 80: 20 \mathrm{v} / \mathrm{v}\right)$, using deionised water (type II) and ethanol p.a. (from Panreac, Barcelona). The alcohol used in the extraction solution and its relative proportion were set to minimise sensor degradation as well as to allow obtain stable potentiometric signals in a minimum time period, avoiding known interferences from other organic solvents, 
Table 1

Sample details of the monovarietal EVOO.

\begin{tabular}{|c|c|c|c|}
\hline $\begin{array}{l}\text { Sample } \\
\mathrm{n}^{\circ}\end{array}$ & Varietal (cvs.) & $\begin{array}{l}\text { Geographical } \\
\text { origin }\end{array}$ & $\begin{array}{l}\text { Production } \\
\text { year }\end{array}$ \\
\hline 1 & Arbequina (ARB) & Spain (Valladolid) & 2011 \\
\hline 2 & Arbequina (ARB) & Spain (Valladolid) & 2011 \\
\hline 3 & Arbequina (ARB) & Spain (Valladolid) & 2011 \\
\hline 4 & Cobrançosa (COB) & $\begin{array}{l}\text { Portugal } \\
\text { (Mirandela) }\end{array}$ & 2011 \\
\hline 5 & Hojiblanca (HOJ) & Spain (Valladolid) & 2011 \\
\hline 6 & Madural (MAD) & $\begin{array}{l}\text { Portugal } \\
\text { (Mirandela) }\end{array}$ & 2011 \\
\hline 7 & Madural (MAD) & $\begin{array}{l}\text { Portugal } \\
\text { (Valpaços) }\end{array}$ & 2011 \\
\hline 8 & Madural (MAD) & $\begin{array}{l}\text { Portugal } \\
\text { (Mirandela) }\end{array}$ & 2011 \\
\hline 9 & Picual (PIC) & Spain (Valladolid) & 2011 \\
\hline 10 & $\begin{array}{l}\text { Verdeal Transmontana } \\
\text { (VER) }\end{array}$ & $\begin{array}{l}\text { Portugal } \\
\text { (Mirandela) }\end{array}$ & 2011 \\
\hline 11 & Arbequina (ARB) & Spain (Valladolid) & 2012 \\
\hline 12 & Arbequina (ARB) & Spain (Valladolid) & 2012 \\
\hline 13 & Arbequina (ARB) & Spain (Valladolid) & 2012 \\
\hline 14 & Cobrançosa (COB) & $\begin{array}{l}\text { Portugal } \\
\text { (Valpaços) }\end{array}$ & 2012 \\
\hline 15 & Hojiblanca (HOJ) & Spain (Valladolid) & 2012 \\
\hline 16 & Madural (MAD) & $\begin{array}{l}\text { Portugal } \\
\text { (Valpaços) }\end{array}$ & 2012 \\
\hline 17 & Picual (PIC) & Spain (Valladolid) & 2012 \\
\hline 18 & $\begin{array}{l}\text { Verdeal Transmontana } \\
\text { (VER) }\end{array}$ & $\begin{array}{l}\text { Portugal } \\
\text { (Valpaços) }\end{array}$ & 2012 \\
\hline
\end{tabular}

such as methanol. Briefly, $10.00 \mathrm{~g}$ of olive oil were vigorously mixed, for $5-10 \mathrm{~min}$, with $100.0 \mathrm{~mL}$ of the hydroethanolic solution and then left to stand for at least $60 \mathrm{~min}$. Then $40.0 \mathrm{~mL}$ of the supernatant solution were removed with an appropriate syringe and immediately analysed using the E-tongue device. Two independent samples were collected from each monovarietal EVOO and analysed in duplicate.

\subsection{E-tongue analysis}

The E-tongue includes two print-screen potentiometric devices, containing different cross-sensitivity membranes as chemical sensors. These were prepared with different pre-established mass combinations of 4 lipidic additives (octadecylamine, oleyl alcohol, methyltrioctylammonium chloride and oleic acid from Fluka; corresponding to approximately 3\%), 5 plasticizers (bis(1-butylpentyl) adipate, dibutyl sebacate, 2-nitrophenyl-octylether, tris(2-ethylhexyl)phosphate and dioctyl phenylphosphonate, from Fluka; representing around 65\%) and high molecular weight polyvinyl chloride (near 32\%). Each sensor was identified with a code with a letter $\mathrm{S}$ (for sensor), followed by the number of the array (1 or 2 ), followed by the number of the membrane (1-20, corresponding to different combinations of plasticiser and additive used).

For each sample the potentiometric signals from the 40 sensors set-up, measured against an $\mathrm{Ag} / \mathrm{AgCl}$ reference electrode (Crison, 5241), were recorded using a multiplexer Agilent Data Acquisition Switch Unit model 34970A controlled with the Agilent BenchLink Data Logger software installed on a PC.

\subsection{Statistical analysis}

A linear discriminant analysis (LDA) together with an SA metaheuristic variable selection algorithm was applied to the E-tongue potentiometric sensor signals recorded. A leave-one-out crossvalidation procedure was used to avoid obtaining an overoptimistic correct classification predictive performance. Subsets of the most informative independent variables (sensors signals) were selected using the SA algorithm, which allowed elimination of redundant variables (sensors) that had a similar contribution to the differences between groups, increasing the accuracy of the prediction. Details concerning the SA algorithm can be found elsewhere (Cadima, Cerdeira, \& Minhoto, 2004; Cadima, Cerdeira, Silva, \& Minhoto, 2012). Bivariate correlations between literature mean phenolic contents of each PT or ES monovarietal EVOO and group centroids of each discriminant cluster, obtained from the potentiometric E-tongue signals, were evaluated using the linear Pearson correlation coefficient. LDA-SA approach was also complemented by multivariate analysis of variance (MANOVA), which is based on general linear model (GLM) procedures for evaluating if olive cultivar, used as a fixed factor (with intra-levels, corresponding to PT and/or ES olive cultivars), had a significant statistical effect (based on the Wilks' Lambda test) on the potentiometric data. The existence of significant statistical differences between the signals potentiometric data, recorded from each sensor, due to olive cultivars was investigated by means of a one-way analysis of variance (one-way ANOVA). When the cultivar effect was statistically significant, the differences between the cultivars were evaluated based on a Tukey's post hoc multiple comparison test. The LDA-SA analysis was performed using the Subselect (Cadima et al., 2004; Cadima et al., 2012) and MASS (Venables, \& Ripley, 2002) packages of the open source statistical program $R$ (version 2.15.1). MANOVA and ANOVA analysis were carried out using JMP Pro 10 software (version 10.0.0). All statistical tests were performed at a significance level of $5 \%$.

\section{Results and discussion}

\subsection{E-tongue signals profiles of EVOO analysis}

On the whole, 72 assays were carried out (18 EVOO samples $\times 2$ extractions of each sample $\times 2$ analyses), each providing 40 potentiometric signals, varying from $-2.0 \mathrm{~V}$ to $+2.0 \mathrm{~V}$ (20 different sensor membranes used in duplicate: $\mathrm{S} 1: 1-\mathrm{S} 1: 20$ and $\mathrm{S} 2: 1-\mathrm{S} 2: 20$ ). Fig. 1 shows the potentiometric signal's box plots for each sensor and each monovarietal EVOO studied, for all the assays carried out, which varied from $-0.05 \mathrm{~V}$ to $+0.60 \mathrm{~V}$ for all sensors included in the E-tongue, avoiding the need for data scaling. Although similar signal profiles were recorded for the hydroethanolic extracts of the monovarietal EVOO studied, slightly intensity differences exist for some sets of sensors, depending on the monovarietal EVOO analysed, which suggest the need to apply a variable selection procedure to choose the most relevant signals for establishing the best predictive LDA model. It should be noted that the differences observed for the signals recorded by the two sensor replicates may be due to slight variations of the membrane composition and physical properties (transparency and porosity) that may occur when a drop-by-drop technique is used for membrane preparation.

\subsection{Portuguese monovarietal EVOO discrimination}

A LDA model with two discriminant functions explaining 100\% of the original data variability ( $99.6 \%$ and $0.4 \%$, respectively) was established based on signals profiles of 18 potentiometric E-tongue sensors (S1:4, S1:7, S1:8, S1:9, S1:11, S1:14, S1:15, S2:3, S2:4, S2:8, $\mathrm{S} 2: 9, \mathrm{~S} 2: 10, \mathrm{~S} 2: 11-\mathrm{S} 2: 15$ and S2:18), selected by the SA algorithm. This variable selection algorithm enabled the inclusion of repeated sensors in the discrimination model ( 6 sensors with the same membrane composition, the remaining 12 having different membrane composition), in agreement with the conclusion of Correia, Magalhães, and Machado (2005), which showed that the inclusion of repeated sensors in arrays for multivariate analysis can improve 
Portuguese monovarietal EVOOs

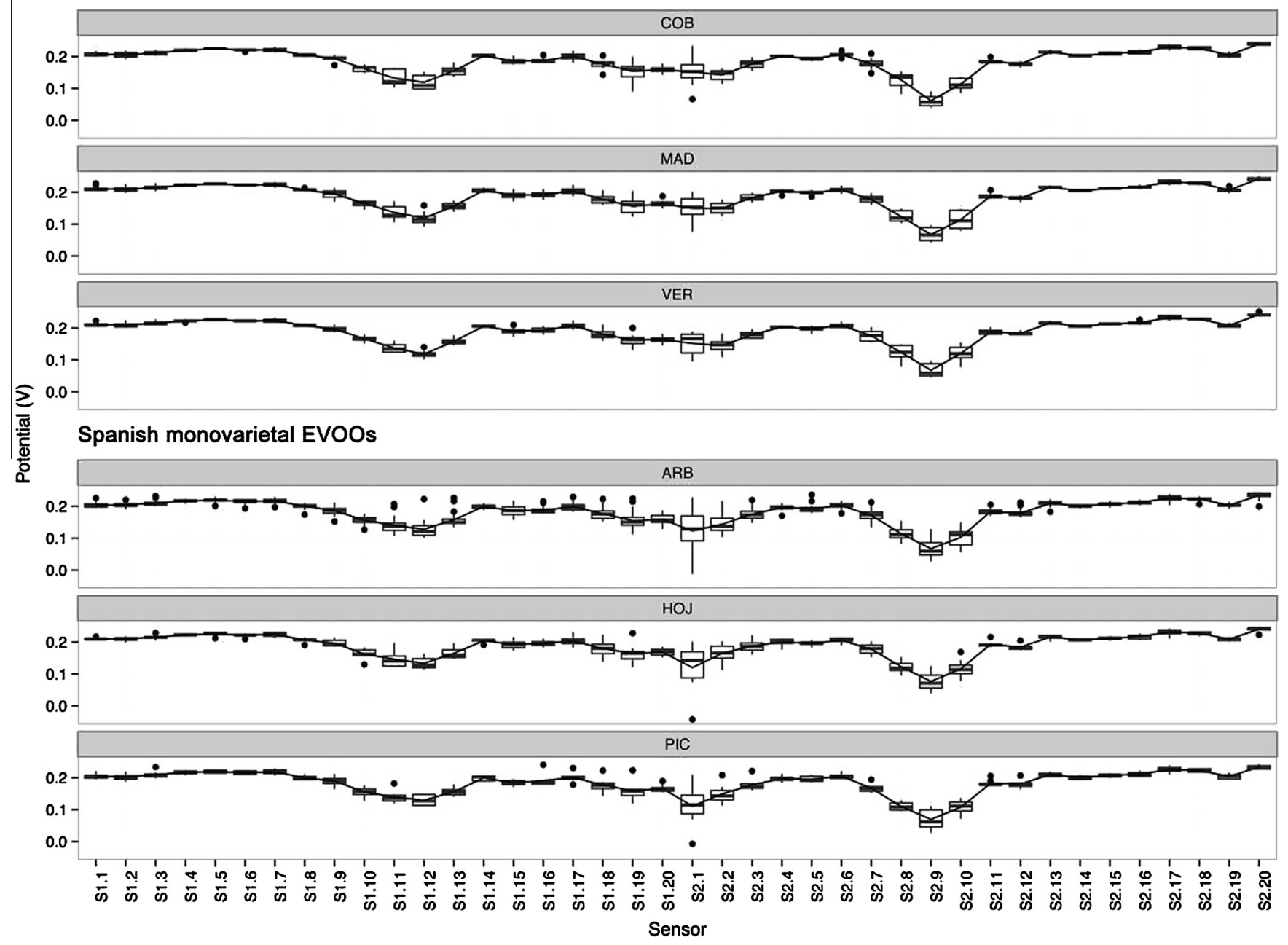

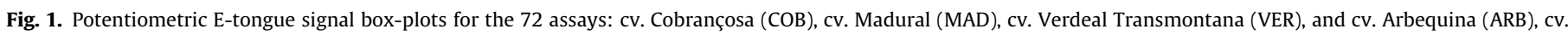
Hojiblanca (HOJ) and cv. Picual (PIC).

the global model performance. The proposed model allowed a $100 \%$ correct classification of the original data (Fig. 2) as well as $100 \%$ predictive correct classification for leave-one-out crossvalidation procedure. This result shows that the E-tongue device could be used to distinguish between Portuguese monovarietal EVOO and so could be used as fast and cost-effective tool to guarantee the correctness of monovarietal EVOO labels from $\mathrm{cV}$. COB, cV. MAD or cv. VER.

The monovarietal EVOO group centroids, which are the mean values of the discriminant scores for a given category of the dependent variable, for the 1st discriminant function (based on the 18 E-tongue sensor signals selected that explained the main data variability), follow the order $\mathrm{COB}<\mathrm{MAD}<\mathrm{VER}$. This tendency may be tentatively related with the nature and amount of the polar compounds in the EVOO extracts. Indeed, Matos et al. (2007) showed that the total contents of tocopherols ( $\alpha-, \beta-$ and $\gamma-)$ and sterols (cholesterol, campesterol, stigmasterol, clerosterol, $\beta$-sitosterol, $\Delta^{5}$-avenasterol and $\Delta^{7}$-avenasterol) in olive oils differ with the olive varietal and the olive maturation index. The means total contents of sterols and tocopherols calculated by Matos et al. (2007) for the same maturation indexes decrease according to $\mathrm{COB} \geqslant \mathrm{MAD} \geqslant$ VER (mean total sterols $(R$-Pearson $=-0.89$ ): $216 \pm 37,209 \pm 56$ and $160 \pm 16 \mathrm{mg} / 100 \mathrm{~g}$ of olive oil, respectively; mean total tocopherols $(R$-Pearson $=-0.94): 248 \pm 11,222 \pm 5$ and

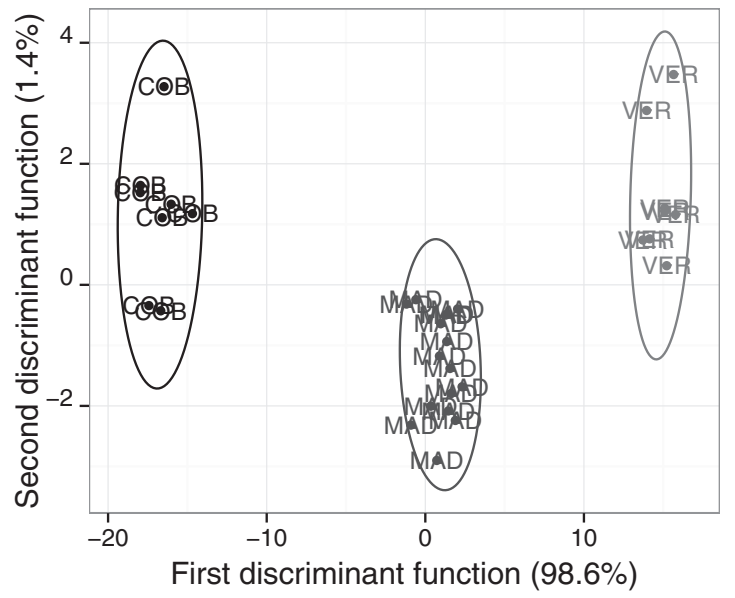

Fig. 2. Portuguese monovarietal EVOO classification performance for the original data: 1st and 2nd LDA functions based on the best E-tongue sensor signal sub-set (18 sensors) selected using the SA meta-heuristic algorithm (COB: cv. Cobrançosa; MAD: cv. Madural; and VER: cv. Verdeal Transmontana).

$144 \pm 6 \mathrm{mg} / \mathrm{kg}$ of olive oil, respectively), showing an inverse tendency when compared with the abovementioned group centroid 
values. This observation suggest that E-tongue signals are inversely related to the contents of sterols and especially of tocopherols in the EVOO hydroethanolic extracts, lower potentiometric signals being registered for higher sterol and particularly tocopherol contents. The fact that the potentiometric signal profiles recorded with the E-tongue could be correlated with the contents of the polar compounds in the EVOO extracts reported in the literature (Matos et al., 2007), which varied considerably with the olive cultivar of the monovarietal EVOO studied (e.g., [tocopherols $]_{\text {COB }}>1.7 \times[\text { tocopherols }]_{V E R}$ ), may justify the drift observed among the EVOO clusters of the LDA plot (Fig. 2). The drift within each cluster may be explained by the fact that monovarietal EVOO have been produced in different years (2011 and 2012, as reported in Table 1) and so affected by different edaphoclimatic conditions, and due to the different storage times of the EVOO until analysis (over one year and less than two months, respectively (although under the same storage conditions, i.e., darkness at $-20^{\circ} \mathrm{C}$ ), which may have influenced the contents of the polar compounds of the EVOO studied.

\subsection{Spanish monovarietal EVOO discrimination}

An LDA model with two discriminant functions explaining $100 \%$ of the original data variability ( $98.4 \%$ and $1.6 \%$, respectively) was established based on signals profiles of 27 of the 40 potentiometric E-tongue available sensors (S1:2, S1:3, S1:5, S1:6, S1:8, S1:9, $\mathrm{S} 1: 12-\mathrm{S} 1: 14, \mathrm{~S} 1: 16-\mathrm{S} 1: 20, \mathrm{~S} 2: 3, \mathrm{~S} 2: 5-\mathrm{S} 2: 9, \mathrm{~S} 2: 11, \mathrm{~S} 2: 12, \mathrm{~S} 2: 14$, $\mathrm{S} 2: 15, \mathrm{~S} 2: 17-\mathrm{S} 2: 19)$, selected using the SA algorithm, which, like in the previous case, included repeated sensors (10 sensors repeated, resulting in the total use of 17 different signals). The proposed model allowed a $100 \%$ correct classification of the original data (Fig. 3) and $97.5 \%$ predictive correct classification for leaveone-out cross-validation procedure, being in this case one of the 24 analyses performed for the cv. ARB classified as cv. HOJ. The very satisfactory global classification predictive performance achieved with the E-tongue can be seen as evidence that the proposed device can be also used to discriminate ES monovarietal EVOO and used as a label authentication tool for cVs. PIC, ARB and $\mathrm{HOJ}$.

Although some studies did not report any statistical difference among sterols or total polyphenols contents for monovarietal EVOO from olives of cvs. ARB, HOJ and PIC (Gorinsteina et al., 2003), different findings were reported by other researchers (Carrasco-Pancorbo et al., 2006; García et al., 2003). García et al.

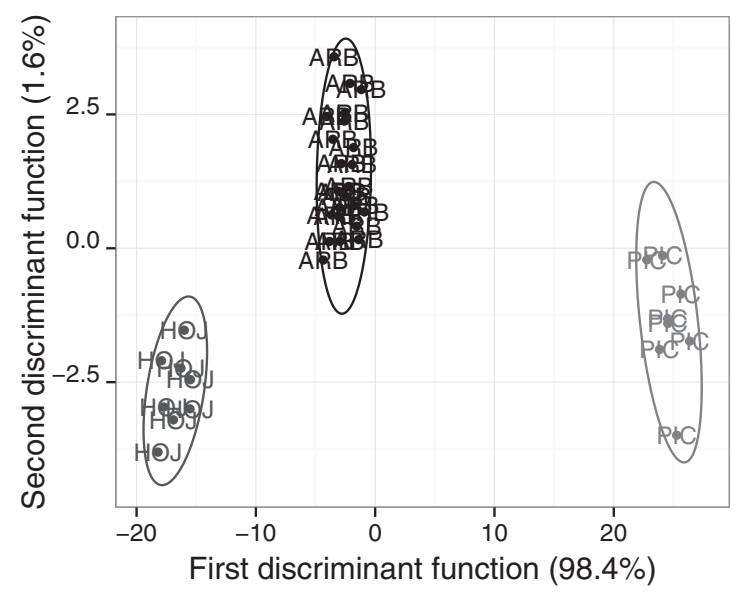

Fig. 3. Spanish monovarietal EVOO classification performance for the original data: 1 st and 2nd LDA functions based on the best E-tongue sensor signals sub-set (27 sensors) selected using the SA meta-heuristic algorithm (ARB: cv. Arbequina; HOJ: cv. Hojiblanca; and PIC: cv. Picual).
(2003) found significant differences for total and individual polyphenol or tocopherol contents, for the same three ES olive cultivars. Indeed, the total polyphenols content (García et al., 2003) followed the order PIC $>$ HOJ $>$ ARB $(1300 \pm 33,1159 \pm 22$ and $1083 \pm 25 \mu \mathrm{mol} / \mathrm{kg}$ of olive oil, respectively), which may justify the fact that in the present work the group centroid of the $\mathrm{cV}$. PIC is located in the positive region for the 1 st linear discriminant function and the cVs. ARB and HOJ group centroids are located in the negative region $(R$-Pearson $=0.77)$. Therefore the 1 st LDA function seems to be related to the total polyphenolic content of the hydroethanolic EVOO extracts. Moreover, the order of the centroids group of this function $(\mathrm{HOJ}<\mathrm{ARB}<\mathrm{PIC}$ ) is in accordance with the increasing mean contents of simple phenols (i.e., tyrosol content $(R$-Pearson $=0.985): 7.3 \pm 0.3,7.7 \pm 0.2$ and $9.5 \pm 0.4 \mathrm{mg}$ / $\mathrm{kg}$ olive oil, respectively) and flavonoids (i.e., luteolin plus apigenin contents $(R$-Pearson $=0.996): 3.2 \pm 0.1,4.0 \pm 0.1$ and $6.3 \pm 0.3 \mathrm{mg} /$ $\mathrm{kg}$ olive oil, respectively); and, with the decreasing content of complex phenols (i.e., secoiridoid form total content $(R$-Pearson $=$ $-0.984): 221 \pm 6,208 \pm 6$ and $145 \pm 4 \mathrm{mg} / \mathrm{kg}$ olive oil, respectively) reported by Carrasco-Pancorbo et al. (2006) for olive oils from cvs. HOJ, ARB and PIC.

On the other hand, the total tocopherols content reported by García et al. (2003) decreased from HOJ $>$ PIC $>$ ARB $(R$-Pearson $=-0.9991 ; 262 \pm 7,238 \pm 12$ and $177 \pm 8 \mathrm{mg} / \mathrm{kg}$ of olive oil, respectively). Therefore, the increasing magnitude of the group centroids of the 2nd LDA function for the ES cultivars (HOJ < PIC < ARB) appear to be inversely related with the total tocopherol content reported by García et al. (2003). In summary, the 2nd LDA function is inversely correlated with the total tocopherol content for the three ES cultivars studied.

Once again, the statistical significant linear correlation between the potentiometric signal profiles recorded with the E-tongue with the literature contents of the polar compounds of the EVOO extracts, which varied considerable with the olive cultivar (e.g., [tocopherols $]_{\mathrm{HOJ}}>1.4 \times$ [tocopherols $_{\mathrm{ARB}} ; \quad$ [flavonoids $]_{\mathrm{PIC}}>1.9 \times$ [flavonoids] $]_{\mathrm{HOJ}}$ ), may justify the drift observed among the EVOO clusters of the LDA plot (Fig. 3). Also, the drift observed within each cluster may be justified by the different edaphoclimatic conditions to which olives were subjected in each year (2011 and 2012, as reported in Table 1) as well as to the different storage times until analysis that may have affected the polar compounds composition of the EVOO.

\subsection{Discrimination between Portuguese and Spanish monovarietal EVOO}

As mentioned before, the monovarietal EVOO under study were produced from different olive cultivars, being 3 of them Portuguese (cvs. COB, MAD and VER), and the other 3 Spanish (cvs. ARB, HOJ and PIC). Although of different geographical origin and specific olive cultivar, the extracted contents and type of polar compounds (e.g., sterols, polyphenols and tocopherols) reported in literature for PT and ES olive cultivars overlap, as can be inferred by analysing the data reported by several researchers (e.g., CarrascoPancorbo et al., 2006; García et al., 2003; Matos et al., 2007). As these compounds are the main components of the hydroethanolic extracts analysed with the E-tongue device, the simultaneous discrimination between these PT and ES monovarietal EVOO may be a difficult task. Indeed, the potentiometric profiles collected in this work did not allow simultaneous discrimination of all the six monovarietal olive oils, contrary to what happened when analysing PT or ES olive oils independently. In fact, the LDA model, which included 26 sensor signals (S1:1-S1:3, S1:8-S1:10, S1:12-S1:14, $\mathrm{S} 1: 16, \mathrm{~S} 1: 18-\mathrm{S} 2: 2, \mathrm{~S} 2: 4-\mathrm{S} 2: 6, \mathrm{~S} 2: 10-\mathrm{S} 2: 12, \mathrm{~S} 2: 14, \mathrm{~S} 2: 15, \mathrm{~S} 2: 17$, S2:18 and S2:20), selected using the SA algorithm, only allowed a 
92\% and $43 \%$ correct classification for original data (Fig. 4) and leave-one-out cross-validation procedures, respectively.

The data presented in Fig. 4 show that in principle the E-tongue device together with the LDA-SA methodology allow splitting of the monovarietal EVOOs studied into two main groups, one containing the PT cultivars located in the 4 th quadrant of the discriminant plot (i.e., positive and negative regions of the 1 st and 2 nd discriminative functions, respectively) and the other with a more broader dispersion, related with the ES cultivars. Nevertheless, the results obtained for the original data group are overoptimistic, allowing a quasi-full discrimination between PT and ES monovarietal EVOO, where none of the PT or ES EVOO was misclassified as belonging to ES or PT cultivars, respectively. However, leave-oneout cross-validation showed that only two EVOO cultivars could be correctly classified according to the country of origin: VER and PIC EVOO were never misclassified as ES or PT EVOO, respectively. A MANOVA followed by one-way ANOVA was also applied for evaluating the relevance of the information contained in the potentiometric signal profiles for distinguishing EVOO according to the olive cultivar. The MANOVA's results allowed us to conclude that olive cultivar had a statistical global significant effect in potentiometric signal profiles ( $p$-value equal to 0.0191 or 0.0020 , using all the 40 sensor signals or the 26 sensor signals selected by SA algorithm as independent variables, respectively). Also, from one-way ANOVA it was shown that olive cultivar had a significant effect on 13 of the 40 E-tongue signal sensors $(0.001 \leqslant p$-value $\leqslant 0.0405$, for $\mathrm{S} 1: 1, \mathrm{~S} 1: 4, \mathrm{~S} 1: 5, \mathrm{~S} 1: 6, \mathrm{~S} 1: 7, \mathrm{~S} 1: 8, \mathrm{~S} 1: 14, \mathrm{~S} 2: 4, \mathrm{~S} 2: 13-\mathrm{S} 2: 15$, $\mathrm{S} 2: 18$ and $\mathrm{S} 2: 20$ ), 8 of these signal sensors being the same as those selected by the SA algorithm as those with more powerful olive cultivar discrimination capability, confirming the variable selection potential of the meta-heuristic algorithm. Indeed, the LDA model based on these 13 sensors selected from the one-way ANOVA had a lower correct classification performance $(61 \%$ and $31 \%$, for original grouped data and leave-one-out cross-validation procedures, respectively) showing that the set of sensors selected using the SA algorithm had a better predictive classification capability. Furthermore, in these cases, and based on the results from the Tukey's post hoc multiple comparison test, cvs. ARB and MAD could be distinguished based on those sensor signals. On the contrary, the signals profiles recorded for the other PT and ES hydroethanolic extracts did not differ significantly ( $p$-value $>0.05$ ), not allowing differentiation of EVOO according to the olive cultivar. Nevertheless, it should be noticed that these conclusions are based on univariate analysis for each sensor, and different results were reached when applying a multivariate combination of sensors data. In fact, the results obtained from LDA-SA (Fig. 4), although partially in accordance with those from univariate analysis, suggest a more easy distinction of $\mathrm{cv}$. PIC from PT olive cultivars than that of $\mathrm{cv}$. ARB.

The performance of the E-tongue device to differentiate each ES cultivar from the three PT cultivars was further evaluated. This task was undertaken since ES olive cultivars have been recently introduced in "Trás-os-Montes" region, which may lead in the near future to a decrease of the importance of PT autochthonous olive cultivars such as cvs. COB, MAD and VER. For this purpose, three studies were carried out trying to infer the E-tongue capability to discriminate each ES monovarietal EVOO (cvs. ARB, HOJ or PIC) from the three Portuguese monovarietal EVOO used (cvs. COB, MAD and VER). For each analysis, a different LDA model was established based on the sensor signals selected by the SA algorithm.

For cv. ARB vs. PT cultivars, three discriminant functions were obtained explaining $100 \%$ of the data variability based on 20 sensor signals, which allowed $89 \%$ and $61 \%$ of correct classifications for original data group (Fig. 5A) and leave-one-out cross-validation procedure, respectively. These less satisfactory results may be partially explained, as already mentioned, by the similarity of the polar compounds contents reported in the literature for cv. ARB and the PT cultivars, as can be inferred by comparing the data concerning for example the tocopherols contents (García et al., 2003; Matos et al., 2007). For cv. HOJ vs. PT cultivars, three discriminant functions were established explaining $100 \%$ of the data variability based on 30 sensor signals, but in this case allowing 100\% and $98 \%$ of correct classifications for original data group (Fig. 5B) and leaveone-out cross-validation procedure, respectively. Finally, for cv. PIC vs. PT cultivars, only two discriminant functions were established

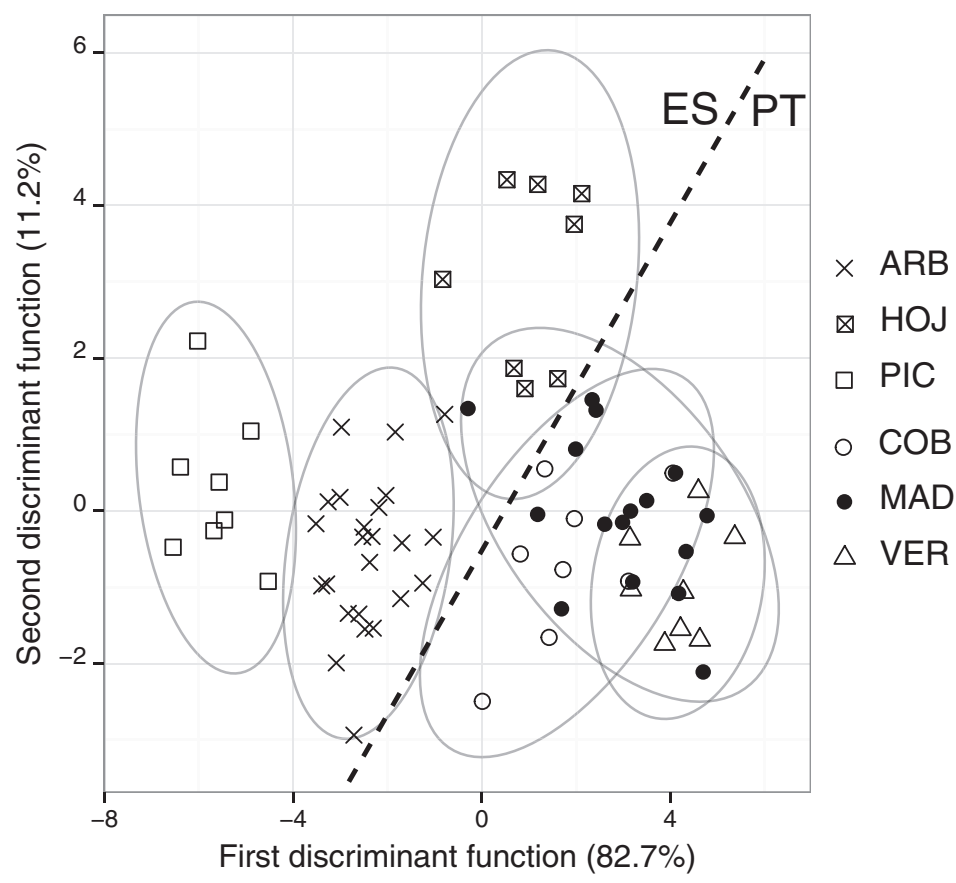

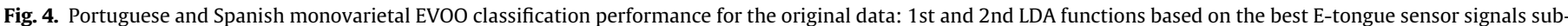

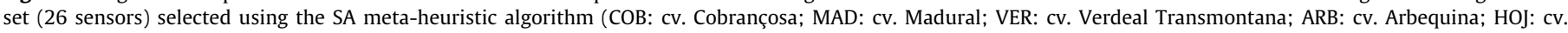
Hojiblanca; and PIC: cv. Picual). 
explaining $100 \%$ of the data variability based on 32 sensor signals allowing $100 \%$ and $93 \%$ of correct classifications for original data group (Fig. 5C) and leave-one-out cross-validation procedure, respectively. Globally, from the LDA, it can be inferred that ARB olive oils have polar contents more similar to those obtained from PT cultivars and, on the other hand, HOJ or PIC olive oils are the most easily distinguished.

Moreover, globally, the cross-validation LOO results also confirmed the satisfactory performance of the E-tongue device, namely concerning the discrimination of Spanish EVOOs of cvs. HOJ or PIC from the monovarietal Portuguese EVOOs. The device never misclassified any of the EVOOs from PT cultivars as cV. HOJ, only one analysis of EVOOs from cv. COB was erroneously
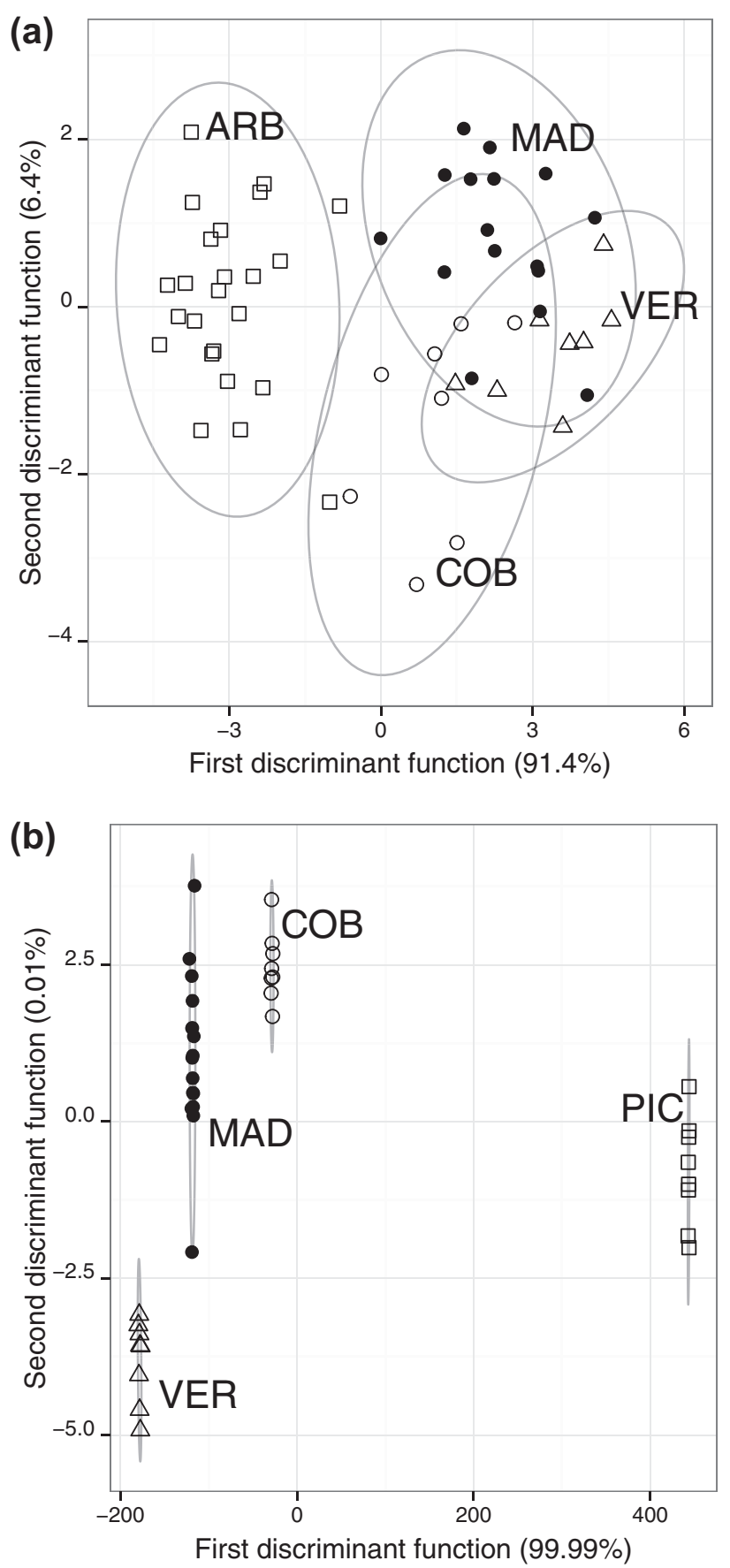

predicted as $\mathrm{cv}$. PIC, two analyses of $\mathrm{cv}$. COB and one of $\mathrm{cv}$. MAD were misclassified as cV. ARB, meaning that only $12.5 \%$ of the PT extracts analysis were misclassified. Finally, none of the EVOOs from cv. VER was classified as ES cultivars, showing that this PT cultivar is the most different one regarding the E-tongue sensor signals profile. On the other hand, only $12.5 \%$ of the EVOO extracts from cVs. PIC, ARB or HOJ were incorrectly predicted as cVs. COB or MAD.

\subsection{Discussion}

From a global point of view, it can be stated that the goodness of the predictive classifications of the six Portuguese and Spanish monovarietal EVOOs, according to olive cultivar, show that:

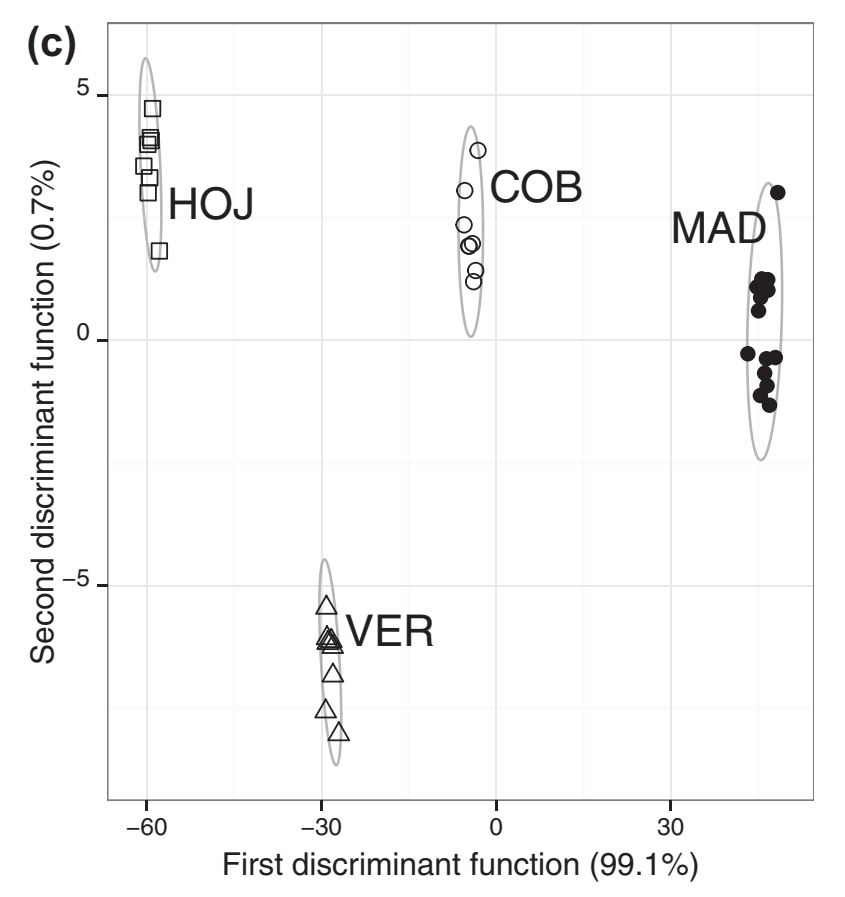

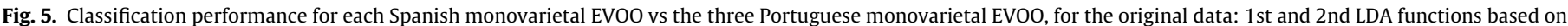

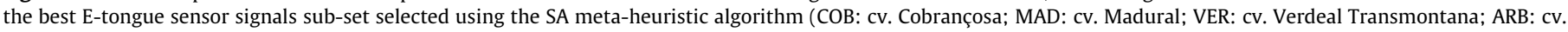
Arbequina; HOJ: cv. Hojiblanca; and PIC: cv. Picual). 
(i) The hydroethanolic extraction solution used turned out to be a feasible choice, providing EVOO extracts representative of each of the six monovarietal olive oil cultivars studied that contained sufficient intrinsic data variability, namely regarding contents of polar compounds, for enabling the subsequent classification tasks.

(ii) The hydroethanolic extraction solution selected ensured the E-tongue stability, avoiding any visible degradation of the duplicate devices and allowing acquiring stable and repeatable sensors signals.

(iii) The meta-heuristic SA variable selection algorithm combined with the LDA methodology, turned out to be a robust tool allowing the selection of the most informative sub-sets of sensors for achieving the best prediction performance.

(iv) The results obtained showed for the first time that lipo-polymeric cross-sensitivity all-solid-state potentiometric E-tongue membranes can be applied to classify and discriminate successfully monovarietal EVOOs according to olive cultivar and geographic origin, with different storage periods but under the same storage conditions (luminosity and temperature).

(v) Finally, the E-tongue clearly distinguished among the three Portuguese monovarietal EVOOs as well as between the three Spanish monovarietal EVOOs. On the other hand it also showed that among the six monovarietal EVOOs studied those of cvs. VER, PIC and HOJ were the most easily differentiated. This could be related to the difference in intensity of the organoleptic characteristics of these olive oils, namely in bitterness, astringency and pungency.

\section{Conclusions}

For the first time and regardless the known difficulties of analysing olive oils with E-tongue due to their non-conductive properties and high viscosity, it was demonstrated that a potentiometric E-tongue had the capability of discriminating monovarietal EVOO, based on the signals profiles recorded for hydro-ethanolic extracts which are richen in polar compounds that are responsible for olive oils bitterness, astringency and pungency. The E-tongue usefulness has been confirmed by analysing and successfully discriminating six monovarietal EVOO (PT cultivars: COB, MAD, VER; and ES cultivars: ARB, HOJ and PIC), with different storage times (from two months to over one year) but stored under the same conditions of light and temperature (darkness at $-20^{\circ} \mathrm{C}$ ) with predictive sensitivities greater than $97.5 \%$. Also, the approach allowed a satisfactory predictive discrimination (61-98\% sensitivity) from each ES monovarietal EVOO and the three PT ones, being EVOO of cVs. VER, HOJ and PIC the olive oils more easily differentiated. Furthermore, for ES and PT discrimination, MANOVA and one-way ANOVA results are in accordance with those achieved with the LDA-SA approach, namely since the potentiometric signal profiles recorded had sufficient information allowing to conclude about the existence of statistical significant cultivar effect, which was mainly due to a set of sensors that globally were the same selected by the SA algorithm and included in the discrimination model.

Nevertheless, it should be emphasised that the proposed device was applied to the analysis of EVOO samples that, although with different storage times until analysis, have been stored under the same conditions and so, the known influence of storage conditions (temperature and light/dark environment) in the organoleptic characteristics of the EVOO studied (Clodoveo et al., 2014; Cosio et al., 2007; Lerma-García et al., 2009) and the possible effect on the potentiometric E-tongue performance was not taken into account, which should be evaluated in future to confirm the device potentiality for monovarietal EVOO discrimination.
In conclusion, the global satisfactory results obtained with the proposed potentiometric E-tongue show that this portable device could be a green, cost-effective and fast alternative tool for EVOO cultivar authentication, enabling the confirmation of the EVOO labels correctness. In addition, the E-tongue can also be seen as useful and helpful complementary tool for olive oil sensory panels.

\section{Acknowledgements}

This work was co-financed by FCT and FEDER under Program COMPETE (Project PEst-C/EQB/LA0020/2013).

\section{References}

Apetrei, I. M., \& Apetrei, C. (2013). Voltammetric e-tongue for the quantification of total polyphenol content in olive oils. Food Research International, 54, 2075-2082.

Apetrei, C., Apetrei, I. M., Villanueva, S., de Saja, J. A., Gutierrez-Rosales, F., \& Rodriguez-Mendez, M. L. (2010). Combination of an e-nose, an e-tongue and an e-eye for the characterisation of olive oils with different degree of bitterness. Analytica Chimica Acta, 663, 91-97.

Apetrei, C., Rodríguez-Méndez, M. L., \& de Saja, J. A. (2005). Modified carbon paste electrodes for discrimination of vegetable oils. Sensors and Actuators B, 111-112, 403-409.

Bakhouche, A., Lozano-Sánchez, J., Beltrán-Debón, R., Joven, J., Segura-Carretero, A., \& Fernández-Gutiérrez, A. (2013). Phenolic characterization and geographical classification of commercial Arbequina extra-virgin olive oils produced in southern Catalonia. Food Research International, 50, 401-408.

Bazakos, C., Dulger, A. O., Uncu, A. T., Spaniolas, S., Spano, T., \& Kalaitzis, P. (2012). A SNP-based PCR-RFLP capillary electrophoresis analysis for the identification of the varietal origin of olive oils. Food Chemistry, 134, 2411-2418.

Cadima, J., Cerdeira, J. O., \& Minhoto, M. (2004). Computational aspects of algorithms for variable selection in the context of principal components. Computational Statistics \& Data Analysis, 47, 225-236.

Cadima, J., Cerdeira, J. O., Silva, P. D., \& Minhoto M. (2012), The subselect R package, (http://cran.r-project.org/web/packages/subselect/vignettes/subselect.pdf).

Cano, M., Roales, J., Castillero, P., Mendoza, P., Calero, A. M., Jiménez-Ot, C., \& Pedrosa, J. M. (2011). Improving the training and data processing of an electronic olfactory system for the classification of virgin olive oil into quality categories. Sensors and Actuators B, 160, 916-922.

Carrasco-Pancorbo, A., Gómez-Caravaca, A. M., Cerretani, L., Bendini, A., SeguraCarretero, A., \& Fernández-Gutiérrez, A. (2006). Rapid quantification of the phenolic fraction of Spanish virgin olive oils by capillary electrophoresis with UV detection. Journal of Agricultural and Food Chemistry, 54, 7984-7991.

Casale, M., Casolino, C., Oliveri, P., \& Forina, M. (2010). The potential of coupling information using three analytical techniques for identifying the geographical origin of Liguria extra virgin olive oil. Food Chemistry, 118, 163-170.

Cimato, A., Monaco, D. D., Distante, C., Epifani, M., Siciliano, P., Taurino, A. M., Zuppa, M., \& Sani, G. (2006). Analysis of single-cultivar extra virgin olive oils by means of an Electronic Nose and HS-SPME/GC/MS methods. Sensors and Actuators B, 114, 674-680.

Clodoveo, M. L., Hbaieb, R. H., Kotti, F., Mugnozza, G. S., \& Gargouri, M. (2014). Mechanical strategies to increase nutritional and sensory quality of virgin olive oil by modulating the endogenous enzyme activities. Comprehensive Reviews in Food Science and Food Safety, 13, 135-154.

Correia, D. P. A., Magalhães, J. M. C. S., \& Machado, A. A. S. C. (2005). Array of potentiometric sensors for simultaneous analysis of urea and potassium. Talanta, 67, 773-782.

Cosio, M. S., Ballabio, D., Benedetti, S., \& Gigliotti, C. (2006). Geographical origin and authentication of extra virgin olive oils by an electronic nose in combination with artificial neural networks. Analytica Chimica Acta, 567, 202-210.

Cosio, M. S., Ballabio, D., Benedetti, S., \& Gigliotti, C. (2007). Evaluation of different storage conditions of extra virgin olive oils with an innovative recognition tool built by means of electronic nose and electronic tongue. Food Chemistry, 101, 485-491.

Dais, P., \& Hatzakis, E. (2013). Quality assessment and authentication of virgin olive oil by NMR spectroscopy: A critical review. Analytica Chimica Acta, 765, 1-27.

Delgado, C., Gómez-Rico, A., \& Guinard, J.-X. (2013). Evaluating bottles and labels versus tasting the oils blind: Effects of packaging and labeling on consumer preferences, purchase intentions and expectations for extra virgin olive oil. Food Research International, 54, 2112-2121.

Escuderos, M. E., Sánchez, S., \& Jiménez, A. (2010). Virgin olive oil sensory evaluation by an artificial olfactory system, based on Quartz Crystal Microbalance (QCM) sensors. Sensors and Actuators B, 147, 159-164.

Escuderos, M. E., Sánchez, S., \& Jiménez, A. (2011). Quartz Crystal Microbalance (QCM) sensor arrays selection for olive oil sensory evaluation. Food Chemistry, $124,857-862$.

García, A., Brenes, M., Garcí, P., Romero, C., \& Garrido, A. (2003). Phenolic content of commercial olive oils. European Food Research Technology, 216, 520-525.

García, A., Brenes, M., Romero, C., García, P., \& Garrido, A. (2002). Study of phenolic compounds in virgin olive oils of the Picual variety. European Food Research Technology, 215, 407-412. 
Garcia, R., Martins, N., \& Cabrita, M. J. (2013). Putative markers of adulteration of extra virgin olive oil with refined olive oil: Prospects and limitations. Food Research International, 54, 2039-2044.

García-González, D. L., \& Aparicio, R. (2004). Classification of different quality virgin olive oils by metal-oxide sensors. European Food Research Technology, 218, $484-487$.

Garrido-Delgado, R., Mercader-Trejo, F., Sielemann, S., de Bruyn, W., Arce, L., \& Valcárcel, M. (2011). Direct classification of olive oils by using two types of ion mobility spectrometers. Analytica Chimica Acta, 696, 108-115.

Gorinsteina, S., Martin-Belloso, O., Katrich, E., Lojek, A., Č́žz, M., Gligelmo-Miguel, N., Haruenkit, R., Park, Y.-S., Jung, S.-T., \& Trakhtenberg, T. (2003). Comparison of the contents of the main biochemical compounds and the antioxidant activity of some Spanish olive oils as determined by four different radical scavenging tests. Journal of Nutritional Biochemistry, 14, 154-159.

Haddi, Z., Alami, H., El Bari, N., Tounsi, M., Barhoumi, H., Maaref, A., JaffrezicRenault, N., \& Bouchikhi, B. (2013). Electronic nose and tongue combination for improved classification of Moroccan virgin olive oil profiles. Food Research International, 54, 1488-1498.

Haddi, Z., Amari, A., Ali, A. O., El Bari, N., Barhoumi, H., Maaref, A., Jaffrezic-Renault, N., \& Bouchikhi, B. (2011). Discrimination and identification of geographical origin virgin olive oil by an e-nose based on MOS sensors and pattern recognition techniques. Procedia Engineering, 25, 1137-1140.

Karabagias, I., Michos, Ch., Badeka, A., Kontakos, S., Stratis, I., \& Kontominas, M. G. (2013). Classification of Western Greek virgin olive oils according to geographical origin based on chromatographic, spectroscopic, conventional and chemometric analyses. Food Research International, 54, 1950-1958.

Lauri, I., Pagano, B., Malmendal, A., Sacchi, R., Novellino, E., \& Randazzo, A. (2013). Application of "magnetic tongue" to the sensory evaluation of extra virgin olive oil. Food Chemistry, 140, 692-699.

Lerma-García, M. J., Simó-Alfonso, E. F., Bendini, A., \& Cerretan, L. (2009). Metal oxide semiconductor sensors for monitoring of oxidative status evolution and sensory analysis of virgin olive oils with different phenolic content. Food Chemistry, 1117, 608-614.

Longobardi, F., Ventrella, A., Napoli, C., Humpfer, E., Schütz, B., Schäfer, H., Kontominas, M. G., \& Sacco, A. (2012). Classification of olive oils according to geographical origin by using ${ }^{1} \mathrm{H}$ NMR fingerprinting combined with multivariate analysis. Food Chemistry, 130, 177-183.
López-Feria, S., Cárdenas, S., García-Mesa, J. A., \& Valcárcel, M. (2008). Classification of extra virgin olive oils according to the protected designation of origin, olive variety and geographical origin. Talanta, 75, 937-943.

Matos, L. C., Cunha, S. C., Amaral, J. S., Pereira, J. A., Andrade, P. B., Seabra, R. M., \& Oliveira, B. P. P. (2007). Chemometric characterization of three varietal olive oils (Cvs. Cobrançosa, Madural and Verdeal Transmontana) extracted from olives with different maturation indices. Food Chemistry, 102, 406-414.

Montealegre, C., Alegre, M. L. M., \& Garcia-Ruíz, C. (2010). Traceability markers to the botanical origin in olive oils. Journal of Agricultural and Food Chemistry, 58, 28-38.

Moore, J. C., Spink, J., \& Lipp, M. (2012). Development and application of a database of food ingredient fraud and economically motivated adulteration from 1980 to 2010. Journal of Food Science, 77, R118-R126.

Morelló, J.-R., Romero, M.-P., \& Motilva, M.-J. (2004). Effect of the maturation process of the olive fruit on the phenolic fraction of drupes and oils from Arbequina, Farga, and Morrut Cultivars. Journal of Agricultural and Food Chemistry, 52, 6002-6009.

Nunes, C. A. (2013). Vibrational spectroscopy and chemometrics to assess authenticity, adulteration and intrinsic quality parameters of edible oils and fats. Food Research International. http://dx.doi.org/10.1016/j.foodres.2013.08.041.

Oliveri, P., Baldo, M. A., Daniele, S., \& Forina, M. (2009). Development of a voltammetric electronic tongue for discrimination of edible oils. Analytical and Bioanalytical Chemistry, 395, 1135-1143.

Pizarro, C., Rodríguez-Tecedor, S., Pérez-del-Notario, N., Esteban-Díez, I., \& González-Sáiz, J. M. (2013). Classification of Spanish extra virgin olive oils by data fusion of visible spectroscopic fingerprints and chemical descriptors. Food Chemistry, 138, 915-922.

Rodríguez-Méndez, M. L., Apetrei, C., \& de Saja, J. A. (2008). Evaluation of the polyphenolic content of extra virgin olive oils using an array of voltammetric sensors. Electrochimica Acta, 53, 5867-5872.

Romero, C., \& Brenes, M. (2012). Analysis of total contents of hydroxytyrosol and tyrosol in olive oils. Journal of Agricultural and Food Chemistry, 60, 9017-9022.

Ruiz-Samblás, C., Tres, A., Koot, A., van Ruth, S. M., González-Casado, A., \& CuadrosRodríguez, L. (2012). Proton tranfer reaction-mass spectrometry volatile organic compound fingerprint for monovarietal extra virgin olive oil identification. Food Chemistry, 134, 589-596.

Venables, W. N., \& Ripley, B. D. (2002). Modern Applied Statistics with S (Statistics and Computing) (4th Edition). New York: Springer. 